\title{
Transposition
}

Musique et Sciences Sociales

Musique : patrimoine immatériel ?

\section{L'après-inscription du gwoka sur la Liste du Patrimoine Culturel Immatériel de l'UNESCO : entretien avec Gabriel Mugerin, Patrick Solvet et Rosan Monza, membres du centre Rèpriz (Guadeloupe)}

\author{
After Gwoka's Inscription on the UNESCO List of the Intangible Cultural \\ Heritage : An Interview with Gabriel Mugerin, Patrick Solvet and Rosan Monza \\ of the Rèpriz Centre (Guadeloupe)
}

\section{Pierre-Eugène Sitchet}

\section{OpenEdition}

\section{Journals}

Édition électronique

URL : http://journals.openedition.org/transposition/4108

DOI : 10.4000/transposition.4108

ISSN : 2110-6134

\section{Éditeur}

CRAL - Centre de recherche sur les arts et le langage

\section{Référence électronique}

Pierre-Eugène Sitchet, "L'après-inscription du gwoka sur la Liste du Patrimoine Culturel Immatériel de I'UNESCO : entretien avec Gabriel Mugerin, Patrick Solvet et Rosan Monza, membres du centre Rèpriz (Guadeloupe) », Transposition [En ligne], 8 | 2019, mis en ligne le 15 octobre 2019, consulté le 16 décembre 2020. URL : http://journals.openedition.org/transposition/4108 ; DOI : https://doi.org/ 10.4000/transposition.4108

Ce document a été généré automatiquement le 16 décembre 2020.

La revue Transposition est mise à disposition selon les termes de la Licence Creative Commons Attribution - Partage dans les Mêmes Conditions 4.0 International. 


\title{
L'après-inscription du gwoka sur la Liste du Patrimoine Culturel Immatériel de l'UNESCO : entretien avec Gabriel Mugerin, Patrick Solvet et Rosan Monza, membres du centre Rèpriz (Guadeloupe)
}

\author{
After Gwoka's Inscription on the UNESCO List of the Intangible Cultural \\ Heritage: An Interview with Gabriel Mugerin, Patrick Solvet and Rosan Monza \\ of the Rèpriz Centre (Guadeloupe)
}

Pierre-Eugène Sitchet

\section{Introduction}

1 Né en Guadeloupe au XVII siècle avec l'esclavage transatlantique, le gwoka est un genre musical qui s'appuie sur le triptyque tambour-chant-danse et accorde une place centrale à la voix. Exclusivement chantée en langue créole, cette musique a été largement oubliée après l'abolition de l'esclavage. Sa renaissance dans le contexte postcolonial tendu des années 1960 comme «musique mémoire» et "musique de résistance » a conduit à sa revitalisation et à sa réappropriation par une grande partie de la population guadeloupéenne.

2 Après avoir créé un festival annuel de gwoka à Sainte-Anne, l'avocat et défenseur de la culture guadeloupéenne Félix Cotellon a fondé en 2005 Rèpriz, le Centre des musiques et danses traditionnelles et populaires de la Guadeloupe. C'est dans ce cadre qu'il a entrepris un ensemble de démarches en vue de l'inscription du gwoka sur la Liste Représentative du Patrimoine Culturel Immatériel (PCI) de l'humanité de l'UNESCO. 
Inscrit sur cette liste depuis le 26 novembre 2014, le gwoka reste aujourd'hui au centre des activités de terrain de Rèpriz.

3 Le présent entretien s'appuie sur les échanges que nous avons eus en août 2018 avec trois des membres du Conseil d'administration ${ }^{1}$ - Gabriel Mugerin, Patrick Solvet et Rosan Monza. Avant de donner la parole à ces trois interlocuteurs acquis au principe de l'inscription du gwoka sur la LR du PCI, il convient de souligner deux éléments de controverse.

4 Si par le passé le gwoka se transmettait confidentiellement et informellement de maître à élève - essentiellement dans le cadre familial -, sa renaissance à la fin du $\mathrm{XX}^{\mathrm{e}}$ siècle, puis en 2014 son inscription sur la LR du PCI, ont entrâné d'importantes modifications, à la fois dans les modalités de sa transmission et dans la diffusion de son répertoire. Importante et complexe, la question de la mise en place de nouveaux modes de transmission et d'une formation officielle des musiciens/praticiens s'est posée et continue de faire débat au sein de la communauté.

5 La seconde source de controverse a trait au bien-fondé ou non de l'inscription ellemême ${ }^{2}$. En effet, certains des débats publics organisés par Rèpriz en vue d'obtenir l'adhésion de la population à la candidature - élément indispensable à la recevabilité du dossier à l'UNESCO - furent houleux. Au point qu'une frange des acteurs du gwoka s'est constituée en collectif pour s'ériger contre une démarche assimilée à une mise sous tutelle de leur musique. Ils estimaient que le gwoka avait "survécu » jusque-là - et qu'il continuerait de vivre par lui-même - sans le soutien d'un quelconque "parrainage ", perçu comme une posture coloniale.

6 Afin de surmonter cet obstacle, les promoteurs du projet s'étaient alors efforcés de développer une réflexion approfondie sur les tenants et les aboutissants de l'obtention d'une reconnaissance internationale par le biais de l'inscription sur la LR du PCI de l'UNESCO de ce genre musical spécifiquement guadeloupéen.

$7 \mathrm{Au}$ fil de l'entretien, Gabriel Mugerin, Patrick Solvet et Rosan Monza abordent l'élaboration du discours concernant le bien-fondé de la démarche menée par Rèpriz auprès de l'UNESCO. Ils évoquent par ailleurs les interrogations suscitées par le concept d'immatérialité, s'agissant d'un patrimoine à première vue bien matériel. Ils nous éclairent ainsi à l'aune de leur compréhension et leur perception de la notion de " patrimoine immatériel » et analysent les enjeux sous-jacents à l'inscription.

8 Gabriel Mugerin, vice-président du Centre Rèpriz depuis juin 2018, est enseignant dans le primaire.

9 Patrick Solvet, membre du conseil d'administration du Centre Rèpriz, est éducateur et animateur.

Rosan Monza (ou Wozan Monza), membre du conseil d'administration du Centre Rèpriz, est chanteur de gwoka et chercheur, docteur en géopolitique de l'Université Paris 8 Vincennes-Saint Denis.

\section{Entretien}


PIERRE-eugène Sitchet : Vous faites tous les trois partie du conseil d'administration du Centre Rèpriz. Pouvez-vous retracer le cheminement personnel qui vous a conduit à vous engager aux côtés de Félix Cotellon?

Gabriel Mugerin : Je suis vice-président de Rèpriz et j'ai la tâche de représenter le président Félix Cotellon, empêché. Je suis enseignant dans le primaire et, sur l'insistance de Patrick ici présent, je me suis tout d'abord engagé dans des activités de terrain au sein du Lyannaj pou Gwoka ${ }^{3}$, collectif étroitement associé à la préparation du dossier en vue de l'inscription du gwoka sur la liste du PCI de l'UNESCO. Après avoir participé activement au volet « communication » au sein du Lyannaj, j'ai intégré Rèpriz et travaillé avec Félix sur les enjeux de l'inscription et les perspectives de l'après-inscription.

C'est dans le cadre de cette collaboration très étroite que lui et moi avons été amenés à réfléchir aux enjeux sous-jacents au projet d'inscription et à élaborer un argumentaire destiné à être présenté aux conseils municipaux des communes de la Guadeloupe, étapes nécessaires pour justifier auprès de l'UNESCO de l'adhésion de la population au projet.

Patrick Solvet : Je suis éducateur de profession et également animateur culturel. Je me considère comme un passeur, non seulement de la musique traditionnelle guadeloupéenne mais de la tradition tout court, que celle-ci soit culinaire, musicale, ou qu'elle relève de savoir-faire. Je m'efforce de transmettre tout cela aux jeunes - $y$ compris les tout petits -, et aux moins jeunes. J'essaye d'établir un pont entre la génération de laquelle j'ai reçu ces traditions et les jeunes générations. Je travaille beaucoup dans les écoles et dans des associations pour contribuer non seulement à la transmission mais aussi à la pérennisation de ces traditions. J'interviens également dans des $\mathrm{EHPAD}^{4}$ et au sein de clubs du troisième âge - où j'essaye de faire revivre à nos aînés ce qu'ils ont eux-mêmes vécu étant jeunes.

Je considère que la musique gwoka est beaucoup plus que de la musique, c'est aussi tout un savoir-vivre, tout un savoir-être. Fort de cette conviction, j'appartenais au CASC de Sainte-Anne ${ }^{5}$ dans le but précis de militer pour assurer au gwoka une forme de pérennité. C'est donc tout naturellement que je me suis engagé dans le Lyannaj pou Gwoka. Un jour Félix Cotellon m'a parlé de sa rencontre avec Chérif Khaznadar, expert du patrimoine culturel immatériel et membre de la Commission nationale française pour l'UNESCO. Peu de temps après, il a évoqué avec moi la possibilité de faire inscrire le gwoka sur la LR du PCI de l'UNESCO. Sur le moment je ne comprenais pas pourquoi. Mais rapidement Félix a su me convaincre. Il a réuni des informations et développé un argumentaire qui m'ont permis de comprendre les tenants et les aboutissants de son idée. La perspective qui s'ouvrait devant nous m'a enthousiasmé et j'ai alors milité bec et ongles pour cette inscription.

La mise en œuvre de ce projet nous a ouvert un grand nombre de portes. Ainsi par exemple dans les écoles, les enseignants étaient en mesure de comprendre l'importance pour les élèves de s'approprier un style musical pouvant prétendre au statut de PCI de l'humanité. Et de ce fait ils sont devenus plus enclins à nous faire intervenir...

Nous ne nous présentions pas seulement en tant que porteurs et transmetteurs de la tradition du gwoka, mais aussi de ce qui dans le gwoka relève du patrimoine culturel immatériel au sens large. Ce qui englobe aussi bien la cuisine, que les vêtements, la démarche corporelle, la façon d'être... Aux yeux des enseignants, l'appropriation par 
les enfants de tous ces éléments culturels élargissait leur bagage culturel et renforçait leurs capacités d'apprentissage. Cela contribuait à les motiver et à les fortifier, à accroître leur fierté.

Aujourd'hui les acteurs culturels de la Guadeloupe peuvent s'emparer de l'inscription du gwoka sur la LR du PCI pour expliquer qu'ils défendent la sauvegarde d'un patrimoine "à valeur universelle exceptionnelle » - à l'instar des acteurs culturels qui défendent la sauvegarde d'un site naturel ou d'un site culturel. Grâce à cette inscription nous, acteurs culturels, disposons désormais de beaucoup plus d'arguments pour faire valoir notre démarche de défense du gwoka. Et cette reconnaissance « booste » nos activités.

Durant la période précédant l'inscription, j'étais membre de la cellule communication du groupe en charge de l'instruction du dossier. Par conséquent, j'intervenais en faveur du gwoka non seulement dans le cadre de mes activités d'animateur culturel mais aussi en direction des médias. Aujourd'hui, je m'investis toujours dans ce type d'activités, notamment envers les personnes qui continuent de manifester leur désaccord à propos de l'inscription. J'essaye de leur faire comprendre l'intérêt de la démarche que nous avons menée. J'explique, encore et encore, les atouts qui découlent de l'obtention du label PCI. J'interviens en particulier dans le milieu associatif, qui a un grand rôle à jouer en tant que porteur de traditions. Ainsi qu'auprès $d u$ grand public. Mon but est de faire prendre conscience que la Guadeloupe possède un patrimoine dont la valeur est si universelle et exceptionnelle qu'il est reconnu comme patrimoine de l'humanité par l'UNESCO. J'explique que c'est à nous tous de le sauvegarder et de le faire connaître. J'interviens aussi dans la mesure du possible auprès des médias, des politiques et des enseignants pour les inciter à transmettre avec pédagogie cette même information via leurs propres réseaux.

Rosan Monza : Je suis chanteur de gwoka, affilié au Lyannaj pou Gwoka et membre du conseil d'administration de Rèpriz. En tant que chanteur de gwoka, j'étais souvent invité au festival annuel de gwoka organisé à l'initiative de Félix Cotellon. C'est dans ces circonstances que je l'ai rencontré et ai développé des liens de solidarité avec les actions qu'il menait. Comme je contribuais aux réflexions menées sur le gwoka en marge de mes activités de chanteur, Félix m'a demandé de venir m'associer au comité qui dans le cadre du Lyannaj réfléchissait à la question du bien-fondé d'une inscription du gwoka sur la LR du PCI de l'UNESCO. Dans la mesure où je pratique le gwoka sur scène, réalise des supports musicaux - CD, DVD -, et compose de la musique, je suis considéré en Guadeloupe comme un musicien de gwoka à part entière. C'est donc en tant que musicien-chanteur de gwoka que j'ai tout d'abord été contacté. Et c'est en m'appuyant sur mon expérience personnelle que je me suis impliqué dans la démarche du Lyannaj.

Au fil du temps, j'ai essayé d'explorer l'aspect plus intellectuel des choses - même si en Guadeloupe ce que recouvre le mot «intellectuel» est parfois considéré avec un peu de mépris. Disons que j'ai essayé de théoriser. Comment structurer une pensée et une conviction pour qu'elles ne soient pas cantonnées uniquement à la gestion d'un patrimoine musical? Comment inscrire la démarche dans la durée ? Comment inscrire celle-ci dans un projet politique? En m'appuyant sur ma formation en géopolitique - j'ai soutenu un doctorat dans cette discipline ${ }^{6}$ - et sur les réflexions 
que j'ai menées sur la Guadeloupe et la guadeloupéanité, je me suis interrogé sur le rôle de cette musique patrimoniale - née il y a près de quatre siècles - dans la construction de l'identité guadeloupéenne.

En premier lieu, je me suis demandé comment le gwoka peut en ce début de XXI siècle contribuer à fixer cette identité et la mettre en partage sous la forme d'un bien commun, tel un marqueur identitaire d'exception. J'ai aussi réfléchi à la façon d'utiliser ce bien commun comme outil de promotion de la culture guadeloupéenne et de l'identité nationale créole - démarche qui relève véritablement du domaine de la géopolitique. La Guadeloupe ne possède ni pétrole, ni capacité d'éclairer le monde, la seule chose que nous puissions apporter à la culture du monde, c'est de mettre en partage ce patrimoine né d'une histoire, d'un rapport de force qui a forgé notre conscience d'appartenance, d'une communauté de destin. Cette musique sert de ferment et de liant à notre société plurielle, une société qui comprend quatre-vingtpour-cent d'Afro-descendants et fait preuve d'une belle capacité à engranger des cultures d'origines différentes - trait qui précisément forge notre guadeloupéanité. Le gwoka est, à l'instar de la langue créole, le substrat, ou le granit, qui construit le peuple de Guadeloupe. C'est autour de cette musique que sont nées les autres musiques ; c'est autour du gwoka que l'intégration des autres cultures musicales s'est faite, que ce soit la biguine, les musiques de traditions blanches, européennes, hindou, indo-guadeloupéennes.

Au-delà de ces réflexions liées à mon parcours personnel, j'avais concrètement au sein du Lyannaj le même rôle que Patrick et Gabriel. On partageait, on échangeait, avec comme objectif premier de faire en sorte que le dossier soit présenté avec suffisamment d'arguments recevables par rapport aux critères exigeants de l'inscription sur la liste du PCI.

On a aussi été amenés à réfléchir au fait que contrairement à d'autres dossiers d'inscription, notre démarche était populaire et démocratique, et qu'elle se confrontait directement au ressentiment existentiel de nombreux guadeloupéens. Il y a eu de vrais débats, et les points de friction et de clivage ont été très importants à un moment donné. Pendant la phase d'instruction du dossier, tout le monde était concerné et tout le monde pouvait donner un avis - extrême ou pas. Le comité du Lyannaj les a tous écoutés et en a fait la synthèse.

Gabriel Mugerin : Je pense qu'il est important d'avoir réussi à croiser les regards des membres de Lyannaj dans ce travail de communication avec la communauté des guadeloupéens. Patrick, représentant les porteurs de tradition, s'est fait courroie de transmission avec l'ONG sur le plan de l'aspect traditionnel du gwoka. Sa légitimité a été mise au service du projet. Quant à Rosan Monza, il a apporté un regard d'universitaire sur la question, notamment sous l'angle de la géopolitique, ce qui a contribué à la validation de la démarche. La dimension politique a d'ailleurs imprégné tout le débat préalable à l'inscription.

Par ailleurs ce croisement des regards a enrichi le dossier proprement dit et a apporté une plus grande légitimité à la démarche : celle-ci était validée à la fois par la population, par les porteurs de tradition et par les universitaires. C'est bien sûr à Félix Cotellon que l'on doit la réunion de ces compétences complémentaires.

Rosan Monza : Oui, on lui doit beaucoup. D'ailleurs qui d'autre qu'un juriste aurait été capable de décrypter le texte de la Convention pour la sauvegarde du PCI, de 
sélectionner les critères à remplir pour que la demande soit recevable, et de suivre avec précision les instructions pour monter le dossier? En outre, bien qu'il ne soit pas lui-même musicien, Félix Cotellon a une connaissance pratique du gwoka. Et c'est un fin observateur qui sait être à l'écoute et collecter les informations pertinentes.

Patrick Solvet : Je voudrais ajouter qu'il est important que Rosan Monza ait été et soit avec nous. Il ne s'en vante pas car il est modeste, mais les gens en Guadeloupe savent qu'il a un doctorat et pour eux ce diplôme universitaire a du poids. Comme par ailleurs il pratique aussi le gwoka, sa présence a apporté une caution lors de la construction du dossier et lui a donné de la crédibilité aux yeux de la société guadeloupéenne. Tout comme il est important qu'il y ait eu des porteurs de tradition professionnellement reconnus qui épousent la cause du PCI.

Je me souviens que certaines personnes ne lisaient pas les tracts que je leur donnais, mais regardaient le nom des gens qui avaient signé. "Ah oui Monza...", "Ah oui Anzala, Erik Cosaque... Alors le projet est ok». Le nom de ces personnalités inspirait confiance aux gens car il était impensable à leurs yeux qu'elles se soient laissées embrigader dans n'importe quoi!

PIERRE-eugène SITCHET: Évoquons maintenant la dialectique entre matérialité et immatérialité, au moment de la démarche de l'inscription et après-inscription.

Gabriel Mugerin : C'est là le cœur du débat. L'argumentaire que nous devions mettre en place pour présenter le projet relevait de cette dialectique. Matériel et immatériel sont des adjectifs qui lorsqu'ils sont employés dans le langage courant le sont parfois à contre-sens. Et il est vrai que lorsque l'on parlait du "gwoka immatériel », cela faisait débat. Le mot gwoka est évocateur de quelque chose de matériel et de tangible: le tambour. C'est pourquoi il a fallu expliquer et clarifier cette notion d'immatériel. Maintenant elle est devenue familière, le mot fait partie du vocabulaire courant dans le monde du gwoka. Mais avant d'en arriver là nous avons été confrontés à la difficulté de rendre la notion d'immatérialité compréhensible à la population, et notamment aux politiques et aux médias.

Patrick Solvet : Cela reste encore une difficulté.

Gabriel Mugerin : Lorsqu'un individu voit les musiciens et le tambour, il a tout simplement l'impression qu'on lui manque de respect quand on vient lui dire que le gwoka est immatériel ; il pense qu'on essaye de lui raconter n'importe quoi... Si bien que nous avons été obligés d'expliquer encore et encore ce que recouvre précisément ce terme. Je me souviens que lors d'une interview à $\mathrm{RCI}^{7}$, Félix Cotellon et moi-même avons été confrontés à l'incompréhension de l'animateur. Patrimoine, ça se réfère à $\mathrm{du}$ solide, non? Alors qu'est-ce que c'est que cette notion de patrimoine immatériel ?... Heureusement, grâce à sa formation de juriste, Félix a su décrypter les termes de la Convention adoptée par l'UNESCO pour la sauvegarde du PCI, ce qui lui a permis d'énoncer dans le langage courant ce que recouvre la notion d'immatérialité. Étape indispensable pour obtenir l'adhésion de la population au projet.

Patrick Solvet : À ce propos, voici un souvenir personnel qu'il me semble intéressant d'évoquer. Lorsque j'intervenais dans les écoles, je demandais aux enfants ce que signifiaient pour eux les adjectifs «matériel » et «immatériel». Un jour l'un d'eux m'a dit : « immatériel, ça veut dire qu'il n'y a rien, et matériel ça veut dire qu'on voit le tambour, le cha cha, il y a ci, il y a ça... ». Je me suis demandé comment expliquer que ce qu'on ne voit pas - ce rien - existe? Avec Félix Cotellon, nous avons 
longuement discuté de cela et sommes arrivés à la conclusion que c'est précisément sur ce point qu'il fallait le plus communiquer. Le challenge était d'expliquer aux enfants qu'ils étaient possesseurs d'une culture immatérielle. Une culture immatérielle à la fois riche de contenu et enrichissante pour celui qui la possède. C'est à ce moment-là qu'on a décidé de se présenter non plus comme « intervenants en musique traditionnelle», mais comme "intervenants en patrimoine culturel immatériel ».

Gabriel Mugerin : Je crois que la difficulté rencontrée à propos de la compréhension de la notion d'immatérialité vient en partie du fait que nous vivons dans une société postcoloniale qui reconnait ses membres au travers de leur patrimoine matériel. Autrement dit, on n'appartient à cette société que si on peut afficher la possession d'un patrimoine matériel. Si bien qu'on ne peut pas dire qu'on a réussi sa vie si on ne possède pas une maison. Ce contexte constitue à mon avis un obstacle à l'appréhension du concept d'une immatérialité qui serait porteuse d'une autre forme de richesse.

Rosan Monza : C'est intéressant pour l'inscription par rapport au patrimoine et c'est intéressant par rapport au ressenti, pas seulement des populations mais aussi en termes de représentation pour la communauté de ce que pourrait être la culture ou the culture, sous-entendu celle qui mérite de la considération. Cela ne concerne pas seulement le peuple. Mais aussi les leaders d'opinion, les politiques, les exécutifs. Ce concept-là n'est pas si immatériel que ça, mais il concerne la partie la plus importante de la culture guadeloupéenne, celle qui forge à la fois l'identité, celle qui est issue de l'atavisme, et qui permet aussi de se projeter et de se singulariser par rapport au reste du monde. C'est cette immatérialité-là qu'il fallait défendre becs et ongles. Il nous fallait à la fois décrypter et décoder le terme juridique, et en même temps ne pas perdre notre fil argumentaire pour que dans la présentation nous respections les exigences de l'inscription telles que définies par l'UNESCO.

Toujours est-il que la notion de "patrimoine immatériel " est un concept très puissant, qui est nouveau, et qui en termes de projection oblige chacun à faire un effort sur soi. Par ailleurs, le peuple guadeloupéen a fait reconnaître que le matériel n'est pas le plus important; que le plus important, c'est ce qui a été transmis.

Gabriel Mugerin : En donnant de la valeur à ce terme «immatériel », on a donné de la valeur à quelque chose qui en Guadeloupe a toujours été dévalorisé. En l'occurrence le gwoka qui a toujours été déconsidéré8. Y compris par les Guadeloupéens eux-mêmes. On a fait en sorte qu'ils se reconnaissent dans quelque chose qu'eux-mêmes ont toujours dévalorisé, et que cette chose devienne un symbole de leur représentativité et de leur identité...

Rosan Monza : Le premier résultat du processus d'inscription a été de déconstruire l'acculturation, ou la fantasmagorie autour de la matérialité, de bousculer la hiérarchie de ce qui est important et de ce qui ne l'est pas.

Gabriel Mugerin : De placer en haut de la hiérarchie des valeurs ce qui précisément en a toujours été rejeté; de montrer que c'est cet «immatériel » qui a de la valeur et qui est le plus représentatif de l'identité guadeloupéenne. Le processus d'inscription a permis de réconcilier les gens avec leur patrimoine et de patrimonialiser ce qu'ils ont toujours rejeté. L'une des difficultés du dossier a été de conjuguer reconnaissance internationale et reconnaissance sur le terrain de ce patrimoine. La première a été 
obtenue après que la seconde a été acquise - après qu'il y a eu consentement libre, éclairé et préalable de la communauté qui sollicitait l'inscription du gwoka. Le fait que le dossier de l'UNESCO décrive le gwoka sous l'intitulé «musique, chants danses et pratique culturelle représentatifs de l'identité guadeloupéenne " précise bien le sens de la démarche, la réconciliation des Guadeloupéens avec leur identité. Nul besoin de continuer à courir après des ombres, leur richesse identitaire se trouve en eux.

Rosan Monza : La matérialité de notre identité, c'est notre immatérialité.

Gabriel Mugerin : Accepter que notre richesse soit immatérielle revient à dire que la Guadeloupe est riche de son patrimoine immatériel. C'est tout le sens du travail qu'il faut continuer à faire - montrer aux gens que ce qu'ils délaissent constitue en fait leur force et se trouve être une source de fierté.

Patrick Solvet: Je me souviens que lorsqu'on a été voir Guy Konkèt ${ }^{9}$ pour lui demander d'adhérer au projet porté/soutenu par Lyannaj, il nous a dit que cela faisait longtemps qu'il appelait à suivre cette voie ; faire entendre la voix du gwoka à l'international, aux Japonais, aux Russes; leur faire entendre le tambour du gwoka. Ajoutant Sé sa ki fos an nou, autrement dit « c'est cela notre force ». Comme il était fier ce jour-là !

PIERRE-eugène Sitchet : Somme toute, que pensez-vous de l'appellation "patrimoine immatériel » ? Le gwoka est-il considéré aujourd'hui comme quelque chose d'immatériel ?

Gabriel Mugerin : Comme je le disais, c'est une appellation provenant d'une instance internationale, une catégorie juridique appliquée à des pratiques très quotidiennes, à une réalité, une façon d'être. Le mois dernier lors de la réunion des États-parties, une des tables rondes portait sur "le patrimoine culturel immatériel dans les droits nationaux ». Lorsque l'on parle de patrimoine immatériel, c'est très important de faire prendre conscience à nos interlocuteurs des différents domaines que recouvre ce patrimoine. En Guadeloupe, il s'agit de la diversité culturelle, et cette diversité culturelle ne relève pas que des chants et des danses. Il existe d'autres domaines qui sont à préserver, et qui sont parfois négligés. Dans le quadrille par exemple, l'accordéon, le siyak, et le tambour de basse sont des éléments menacés. De ce fait, on peut avoir l'impression que "patrimoine immatériel» est une expression qui regroupe musique, chant et danse traditionnelle. Or notre patrimoine immatériel est beaucoup plus vaste que cela. Il inclut aussi bien la connaissance des plantes que la langue elle-même, les fêtes religieuses, tout ce qui singularise notre communauté. Mais les gens ont du mal à la définir cette notion de communauté d'un point de vue politique. Ils pensent que l'on parle de Guadeloupe. On ne parle pas de Guadeloupe, on parle de personnes qui sont à l'œuvre en Guadeloupe et d'un élément qui représente la Guadeloupe.

PIERRE-eugène SITCHet : Quelles sont les répercussions en Guadeloupe de l'inscription du gwoka au PCl, de cette sorte de labellisation UNESCO ?

Rosan Monza : L'inscription au PCI ne constitue pas une « labellisation » UNESCO.

Gabriel Mugerin : Nous avons obtenu une reconnaissance internationale du gwoka, pas un label.

Patrick Solvet : Par contre il existe un label UNESCO que n'importe qui peut solliciter pour un projet culturel qui défend une cause. 
Gabriel Mugerin : Nous avons d'ailleurs demandé et obtenu ce label pour le film documentaire ${ }^{10}$ que Philippe Mugerin a tourné en Guadeloupe pendant la préparation du dossier et à Paris le 26 novembre 2014, jour de l'inscription au PCI. Ce documentaire bénéficie d'une estampille avec le logo de l'UNESCO. Mais en aucun cas la procédure d'inscription ne peut être assimilée à une labellisation. À Rèpriz nous avons passé beaucoup de temps à préciser que l'inscription sur la Liste du PCI n'était pas un label!

PIERRE-eUgène SITCHeT : Merci pour ces précisions. J'ajuste ma question : quelles sont en Guadeloupe les répercussions et l'impact politique de l'inscription au PCI ? Notamment sur les acteurs du gwoka.

Gabriel Mugerin : L'impact politique passe tout d'abord par le changement de statut du gwoka. Cela se perçoit parce qu'on parle plus du gwoka qu'auparavant. Il y a une appropriation de la notion de patrimoine immatériel. On entend cette expression dans la bouche de personnes qui n'en avaient jamais entendu parler auparavant. Il reste maintenant à sensibiliser les responsables politiques à la mise en œuvre des engagements pris dans le livret rédigé lorsque nous sommes allés à leur rencontre. Un grand nombre d'entre eux étaient présents à Paris le jour de l'inscription sur la Liste du PCI, le 26 novembre 2014, parce que le congrès des maires avait lieu en même temps ${ }^{11}$. Ils ont signé sous le coup de l'émotion. Beaucoup de ceux qui témoignent dans le film n'ont pas - ou pas encore - donné suite à l'engagement qu'ils ont pris ce jour-là. Il est donc important de continuer les actions de sensibilisation maintenant que le statut du gwoka a changé, et qu'il relève désormais du patrimoine culturel immatériel. Nous disons aux politiques qu'il faut passer à l'action et faire ce qu'on a dit qu'on allait faire.

Patrick Solvet : Les politiques en restent souvent aux mots.

Rosan Monza : Il y a plus que les mots tout de même. Quand on dit que le gwoka est un patrimoine culturel immatériel, cela signifie qu'en cas de contentieux avec une collectivité ou une administration, il existe des textes auxquels on peut se référer. À l'instar des codes des marchés publics, des codes de l'urbanisme, ou bien des codes de passation des marchés. La sauvegarde du gwoka relève maintenant de l'État qui doit régulièrement présenter des rapports à l'UNESCO.

Gabriel Mugerin : Par ailleurs de nouvelles contraintes figurent dans le cadre des Directives opérationnelles ${ }^{12}$ de l'UNESCO, notamment pour la sauvegarde du patrimoine immatériel dans les situations de crise. Cela implique par exemple l'obligation d'inscrire l'inventaire du patrimoine immatériel dans les plans de prévention des risques.

PIERRE-eugène Sitchet : Quels sont les objectifs que le Centre Rèpriz s'est fixé pour les années à venir tant dans le domaine de la transmission et de l'enseignement artistique que pour la sauvegarde du gwoka désormais patrimoine culturel immatériel ?

Gabriel Mugerin : Le premier axe est effectivement la sauvegarde, la mise en œuvre du mot «sauvegarder». Autrement dit, assurer la viabilité dans la durée. En collectant de la documentation au sens large, et en assurant la pratique du patrimoine vivant et sa transmission.

En ce qui concerne la documentation, on essaye de faire comprendre aux conseils municipaux l'importance de réaliser l'inventaire du patrimoine immatériel à leur échelle. Pas seulement l'inventaire des praticiens du gwoka, pas seulement de ceux 
du quadrille, l'autre danse la plus en vue en Guadeloupe. Mais aussi l'inventaire des autres acteurs, y compris de ceux qui ne sont plus pratiquants. C'est possible par le bouche-à-oreille. Une telle collecte documentaire peut permettre de communiquer sur le gwoka, sur le patrimoine, sur le territoire... Comme nous l'avons dit précédemment, il y a eu le film de Philippe Mugerin.

Quant à la transmission, c'est au niveau des écoles qu'on souhaiterait qu'elle se fasse. Avec les porteurs de traditions, ce qui constitue l'une des difficultés. Patrick est bien placé pour le savoir. À présent tout le monde peut faire du créole, y compris quelqu'un qui ne connaît pas le créole, puisqu'on ne parle plus de la langue et de la culture créoles mais... de créole langue vivante. Si aujourd'hui, comme pour le créole, tout le monde se mettait à faire du gwoka sans l'appui des porteurs de traditions, il y aurait un problème. Par exemple, des conflits de légitimité. Personnellement je suis enseignant, je peux enseigner ce qui est dans les programmes, j'ai donc le droit d'enseigner le gwoka. Mais aux yeux de Patrick Solvet, ka misyé konnèt an gwoka ${ }^{13}$. En l'occurrence, il ne s'agit pas d'enseigner la technique gwoka, il s'agit d'enseigner le patrimoine gwoka. Or pour ce faire, il faut disposer de documents didactiques. Et il faut que ceux-ci soient conçus de telle sorte que deux personnes, l'une en faveur de l'inscription, l'autre contre, puissent se reconnaître dans le même document. Il n'est pas question que chacun transmette «sa » vérité.

Par exemple nous avons entrepris une collecte documentaire sur Vélo ${ }^{14}$ parce que ce chanteur constitue un élément du patrimoine immatériel gwoka. Des gens qui ne l'ont pas du tout connu nous ont dit : sété boug an mwen ${ }^{15}$. Quand on recoupe leur âge avec le sien, ils avaient trois ans quand ils étaient « son pote »! (Rires) Aujourd'hui ils en ont quarante et prétendent qu'ils ont fait du gwoka avec lui! Tout cela pour montrer l'importance du travail scientifique autour du gwoka. Le travail scientifique est un préalable à la transmission.

Rosan Monza : Revenons à la transmission et à la pédagogie. Comment transmettre? Que transmettre? Si l'on inclut des éléments très peu vérifiables scientifiquement, ce sera la porte ouverte à toutes les dérives et les extrémismes. Je pense qu'il faut être très vigilant. Dans une de mes chansons, je parlais de la nécessité absolue d'avoir une spiritualité fondale ${ }^{16}$, provenant de sa propre expérience. En d'autres termes, chacun expérimente individuellement sa relation avec le Gwoka. À titre personnel, le gwoka m'a procuré des forces, il m'a donné des choses que je ne saurais pas expliquer à d'autres. Loin de moi l'idée de faire du prosélytisme, même si chacun a le droit d'expérimenter. Il ne faut pas prendre le risque de fermer les portes aux pédagogues, à tous ceux qui travaillent sur la transmission, sur la didactique, sur comment transmettre, comment bien transmettre. Il ne faut pas prendre le risque de mélanger les genres. Toutefois, au stade de cette discussion, j'entrevois un point de conflit qui risque d'apparaître un jour. Il s'agit de la dimension sacrée du gwoka. Dimension qui n'a pas été prise en compte, qu'on a "savamment» évacuée, parce que Félix était quelque part garant d'une certaine rationalité, de la scientificité de la démarche pour la reconnaissance et l'identification du gwoka.

Gabriel Mugerin : Je suis persuadé qu'il y a une dimension sacrée dans la musique et qu'il y a une énergie de cet ordre dans le gwoka. Cependant il ne faut pas faire croire que cela est incantatoire. Il faudrait se mettre d'accord sur ce que signifie ce côté mystique ou sacré qui est présent dans le Gwoka. Il faudrait se mettre d'accord sur 
des règles pour transmettre cet aspect-là du gwoka. Mais comment faire? Tout le monde n'a pas atteint le même niveau d'initiation. Et qui finalement maîtrise cette dimension sacrée du gwoka?

PIERRE-eUgène SITCHET : Nous constatons qu'il reste encore beaucoup de questions en suspens. Je vous remercie d'avoir témoigné des multiples enjeux de cette inscription et de sa viabilité en termes de sauvegarde une fois la reconnaissance de l'UNESCO obtenue.

\section{NOTES}

1. Félix Cotellon, empêché, n'a malheureusement pas pu participer à l'entretien.

2. Les aspects de cette controverse sont amplement développés dans la contribution de Dominique Cyrille et Florabelle Spielmann à ce dossier thématique.

3. En créole lyannaj signifie "unir les forces», ou encore "l'union fait la force». Lyannaj pou Gwoka est le nom du collectif créé par les partisans de la défense de la cause du gwoka par le biais de la procédure d'inscription.

4. Établissements d'Hébergement pour Personnes Âgées Dépendantes.

5. Comité d'Animations Sportives et Culturelles de la commune de Sainte-Anne, Guadeloupe.

6. MONZA Rosan, La Guadeloupe : kaléidoscope de la géopolitique d'un département français de la Caraïbe, thèse de doctorat en géopolitique sous la direction d'Yves Lacoste, Université Paris 8 VincennesSaint Denis, 1996.

7. Radio Caraïbes International.

8. À ce sujet, voir la contribution de Dominique Cyrille et Florabelle Spielmann au présent dossier thématique.

9. Guy Konkèt (1950-2012) est un éminent chanteur de gwoka.

10. MUGERIN Philippe (réalisateur), Gwoka-la linèsko, jou mèkrédi-lasa, Centre Rèpriz, 2015, 41 minutes.

11. Du 25 au 27 novembre 2014, Porte de Versailles.

12. Il s'agit de l'édition 2016 des Directives opérationnelles pour la mise en œuvre de la Convention pour la sauvegarde du patrimoine culturel immatériel.

13. «Que connait-il du gwoka?»

14. Marcel Lollia (1931-1984), dit Vélo, est le maître tanbouyé le plus célèbre et le plus glorifié de l'archipel guadeloupéen.

15. «C'était mon pote. »

16. Mot utilisé en créole qui veut dire profond et ancestral. Il se rapporte à la notion de fondement. 


\section{RÉSUMÉS}

Fondé par Félix Cotellon en 2005, Rèpriz, le Centre des musiques et danses traditionnelles et populaires de la Guadeloupe, a mené un ensemble d'actions conduisant à l'inscription du gwoka sur la Liste Représentative du Patrimoine Culturel Immatériel (PCI) de l'humanité de l'UNESCO en 2014.

Le présent entretien s'appuie sur un échange avec trois membres du conseil d'administration de Rèpriz: Gabriel Mugerin, Patrick Solvet et Rosan Monza, au gré duquel ils reviennent sur l'élaboration du discours qui a permis d'expliquer à la communauté le bien-fondé de leur démarche auprès de l'UNESCO. Ils évoquent par ailleurs les interrogations suscitées par le concept d'immatérialité s'agissant d'un patrimoine à première vue bien matériel. Ils nous éclairent ainsi à l'aune de leur compréhension de la notion de "patrimoine immatériel ", et analysent les enjeux sous-jacents à l'inscription ainsi que ses effets observés quatre ans plus tard.

Founded by Félix Cotellon in 2005, Rèpriz, the Centre for Traditional and Popular Music and Dances of Guadeloupe, conducted a series of actions leading to the inscription of gwoka music on UNESCO's Representative List of the Intangible Cultural Heritage (ICH) of Humanity in 2014.

This interview is based on a conversation with three Rèpriz board members, Gabriel Mugerin, Patrick Solvet and Rosan Monza, in which they recall the process of determining the discourse used to explain the legitimacy of their approach with UNESCO to the community. They also address questions raised by the concept of intangibility in the case of heritage that is at first sight very tangible. They shed light on their understanding of the notion of "intangible heritage" and examine underlying issues with regard to the inscription and its effects observed four years later.

\section{INDEX}

Keywords : intangible cultural heritage, Guadeloupe, gwoka, UNESCO, West Indies

Mots-clés : patrimoine culturel immatériel, Guadeloupe, gwoka, UNESCO, Antilles

\section{AUTEUR}

\section{PIERRE-EUGÈNE SITCHET}

Franco-américain d'origine camerounaise, Pierre-Eugène Sitchet (aka Gino Sitson) est vocaliste et compositeur (huit albums solo), et chercheur en musicologie. Ses recherches portent essentiellement sur la cognition musicale, les propriétés expressives de la voix et les processus de transmission des musiques de la diaspora « noire ». Parmi celles-ci, il s'intéresse tout particulièrement au gwoka. Docteur en musicologie de l'Université Paris-Sorbonne, il a notamment dispensé des cours à Columbia University (New York), Florida Memorial University (Miami), Long Island University (New York), Queens College (New York), University of Panama City (Panama). En 2006, il s'est vu décerner le titre de Cultural Arts Ambassador de Miami (Floride) et celui de citoyen d'honneur de cette ville. Depuis 2016, il est Ambassadeur de bonne volonté du Fonds des Nations Unies pour l'enfance (UNICEF-Cameroun). 